\title{
Serum or plasma cartilage oligomeric matrix protein concentration as a diagnostic marker in pseudoachondroplasia: differential diagnosis of a family
}

\author{
A Cevik Tufan*,1,2,7, N Lale Satiroglu-Tufan ${ }^{2,3,7}$, Gail C Jackson ${ }^{4}$, C Nur Semerci ${ }^{3}$, \\ Savas Solak ${ }^{5}$ and Baki Yagci ${ }^{6}$
}

\begin{abstract}
${ }^{1}$ Department of Histology and Embryology, School of Medicine, Pamukkale University, Denizli, Turkey; ${ }^{2}$ Pamukkale University Research Center for Genetic Engineering and Biotechnology, Denizli, Turkey; ${ }^{3}$ Molecular Genetics Laboratory, Department of Medical Biology, Center for Genetic Diagnosis, School of Medicine, Pamukkale University, Denizli, Turkey; ${ }^{4}$ NGRL, St Mary's Hospital, Manchester, UK; ${ }^{5}$ Birgi Medical Center, Republic of Turkey Ministry of Health, Izmir, Turkey; ${ }^{6}$ Department of Radiology, School of Medicine, Pamukkale University, Denizli, Turkey
\end{abstract}

Pseudoachondroplasia (PSACH) is an autosomal-dominant osteochondrodysplasia due to mutations in the gene encoding cartilage oligomeric matrix protein (COMP). Clinical diagnosis of PSACH is based primarily on family history, physical examination, and radiographic evaluation, and is sometimes extremely difficult, particularly in adult patients. Genetic diagnosis based on DNA sequencing, on the other hand, can be expensive, time-consuming, and intensive because COMP mutations may be scattered throughout the gene. However, there is evidence that decreased plasma COMP concentration may serve as a diagnostic marker in PSACH, particularly in adult patients. Here, we report the serum and/or plasma COMP concentration-based differential diagnosis of a family with affected adult members. The mean serum and/or plasma COMP concentrations of the three affected family members alive $(0.69 \pm 0.15$ and/or $0.81 \pm 0.08 \mu \mathrm{g} / \mathrm{ml}$, respectively) were significantly lower than those of an age-compatible control group of 21 adults $(1.52 \pm 0.37$ and/or $1.37 \pm 0.36 \mu \mathrm{g} / \mathrm{ml}$, respectively; $P<0.0001)$. Bidirectional fluorescent DNA sequencing-based genetic diagnosis of these patients revealed a heterozygous mutation for the nucleotide change $1532 \mathrm{~A}>\mathrm{G}$ in exon 14 of the COMP gene, resulting in a substitution of amino acid 511 from aspartic acid to glycine in COMP. Thus, serum and/or plasma COMP concentration may be suggested as an additional diagnostic marker to aid clinical and radiographic findings in suspected cases of PSACH. European Journal of Human Genetics (2007) 15, 1023-1028; doi:10.1038/sj.ejhg.5201882; published online 20 June 2007

Keywords: pseudoachondroplasia; cartilage oligomeric matrix protein; mutation; diagnosis; serum; plasma

\footnotetext{
*Correspondence: Dr AC Tufan, Department of Histology and Embryology, School of Medicine, Pamukkale University, Kinikli Kampusu, Morfoloji Binasi, Kat-1, Kinikli, Denizli, Turkey.

Tel: + 90258295 2472; Fax: + 90258295 2433;

E-mail: actufan@pau.edu.tr

${ }^{7}$ These authors contributed equally to this work.

Received 14 February 2007; revised 28 April 2007; accepted 2 May 2007; published online 20 June 2007
}

\section{Introduction}

Pseudoachondroplasia (PSACH; MIM 177170) is an autosomal-dominant osteochondrodysplasia affecting at least 1 in 20000 individuals and is characterized by disproportionate short stature, deformity of the lower limbs, short fingers, loose joints, and ligamentous laxity. ${ }^{1-3}$ Other characteristic features include normal craniofacial appearance and early-onset osteoarthritis. Its radiological findings include small, irregular epiphyses and irregular, mush- 
roomed metaphyses of all tubular bones and flattening and/or anterior beaking of vertebrae. Adult stature has been reported as $82-130 \mathrm{~cm}$. Although the vertebral changes resolve with age (ie, after puberty, the vertebrae become more normal in appearance), the epiphyseal changes of the long bones become more severe, leading to progressive degeneration and severe osteoarthritis.

PSACH is caused exclusively by mutations in the cartilage oligomeric matrix protein (COMP) gene located on chromosome 19p13.1, and, to date, all cases of PSACH have been ascribed to mutations in exons 8-19 of this gene. ${ }^{4}$ COMP is a homopentameric glycoprotein found in cartilage, tendon, ligament, smooth muscle, synovium, and osteoblast. ${ }^{5,6}$ COMP is described as a key component of the extracellular matrix (ECM) of the skeleton. COMP binds collagen types I, II, and IX through its C-terminal globular domain, ${ }^{7-9}$ forming a large structural network of macromolecules in the ECM. The secretion of mutant COMP from chondrocytes is markedly delayed, presumably due to a prolonged association with chaperones in the rough endoplasmic reticulum (rER). ${ }^{10}$ Thus, PSACH appears to be an rER storage disease most likely caused by improper folding of mutant COMP. The growth failure of patients with PSACH may be explained by an increased cell death of growth plate chondrocytes.

Current clinical diagnosis of PSACH is based primarily on family history, physical examination, and radiographic evaluation. However, clinical presentation varies substantially between individual cases and depends on the patients' age. Most radiographic changes are not disease-specific, leading to misdiagnosis, particularly in adult patients. ${ }^{11}$ It has been reported that the characteristic findings of the spine resolves with time and normalizes in adolescence, making diagnosis even more difficult. ${ }^{12}$ In addition, radiological diagnosis is more difficult in adulthood owing to associated changes resulting from osteoarthritis. ${ }^{11}$ Genetic diagnosis based on DNA sequencing, on the other hand, can be expensive and labor-intensive because COMP mutations can be scattered throughout the gene containing 19 exons. ${ }^{4}$ However, there is evidence that decreased plasma COMP concentration may serve as a diagnostic marker in $\mathrm{PSACH}$, particularly in adult patients. ${ }^{12}$ Mabuchi et $a l^{12}$ presented evidence that circulating COMP is decreased in PSACH patients carrying COMP mutations compared with controls.

Here, we report the serum and/or plasma COMP concentration-based differential diagnosis of a family with affected adult members in two generations. All the three affected members alive were adults with noncharacteristic radiological and clinical features.

\section{Materials and methods Case history}

The subjects are the three dwarf members (individuals II-4, II-6, and III-1) of a family (see pedigree in Figure 1). The

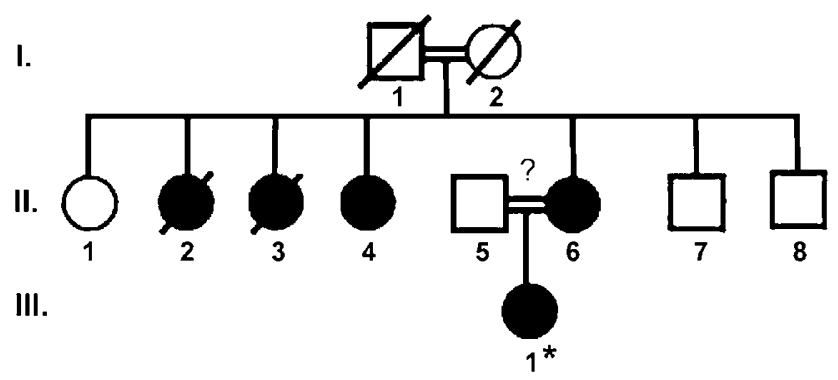

Figure 1 Family pedigree. Unnecessary spouses were omitted from the original pedigree to simplify the presentation of the family. Filled circles, pseudoachondroplasia (PSACH); unfilled circles and squares, unaffected; $\left({ }^{*}\right)$, proband.

grandparents of the proband (individual III-1) were first cousins to each other. They have had five daughters, of whom four of them were affected, and two healthy sons. Two of the four affected daughters (individuals II- 2 and II3 ) died at ages 78 and 45, respectively. Individual II-4 is one of the two affected daughters alive. She is 80 years old, $100 \mathrm{~cm}$ in height, and suffers from severe osteoarthrosis. Individual II-6, the other affected-daughter alive, on the other hand, is 60 years old, $90 \mathrm{~cm}$ in height, and the mother of the proband. She also suffers from severe osteoarthrosis. The proband, 36 years old and $119 \mathrm{~cm}$ in height, is the only daughter of individual II- 6 from a possibly consanguineous marriage to individual II-5. She is an intelligent woman with normal craniofacial appearance. A short stature, disproportionately short limbs, and short fingers are her only known medical problems (Figure 2). Her radiological skeletal survey showed shortening of long bones and widening in her epiphyses (Figure 3). Phalanges, metacarpals, and metatarsals were short and thick with cone-shaped epiphyses (Figure 3a and d). Chest radiogram showed superiorly curved clavicles and spatulate ribs with marked costochondral calcifications (Figure 3c). Lateral radiograph of the spine revealed straightening of dorsal kyphosis with decreased anteroposterior diameter of the chest and small central endplate depressions of some dorsal vertebrae (Figure $3 \mathrm{~b}$ ). There was normal widening of the interpedicular distances from upper to lower spine (Figure 3b). Pelvis radiogram showed flattened, irregular, and fragmented femoral heads with shortened necks, increased acetabular angles, and widened symphysis pubis (Figure 3e). The skull was normal.

These adulthood findings were uncharacteristic of a given osteochondrodysplasia, but were suggestive of PSACH or Acromesomelic dysplasia, Maroteaux type (AMDM, MIM 602875). The review of this family by the European Skeletal Dysplasia Network (ESDN) supported our diagnosis (Case number ESDN-00490) and suggested COMP mutation analysis as the first choice of action for the definitive diagnosis. 


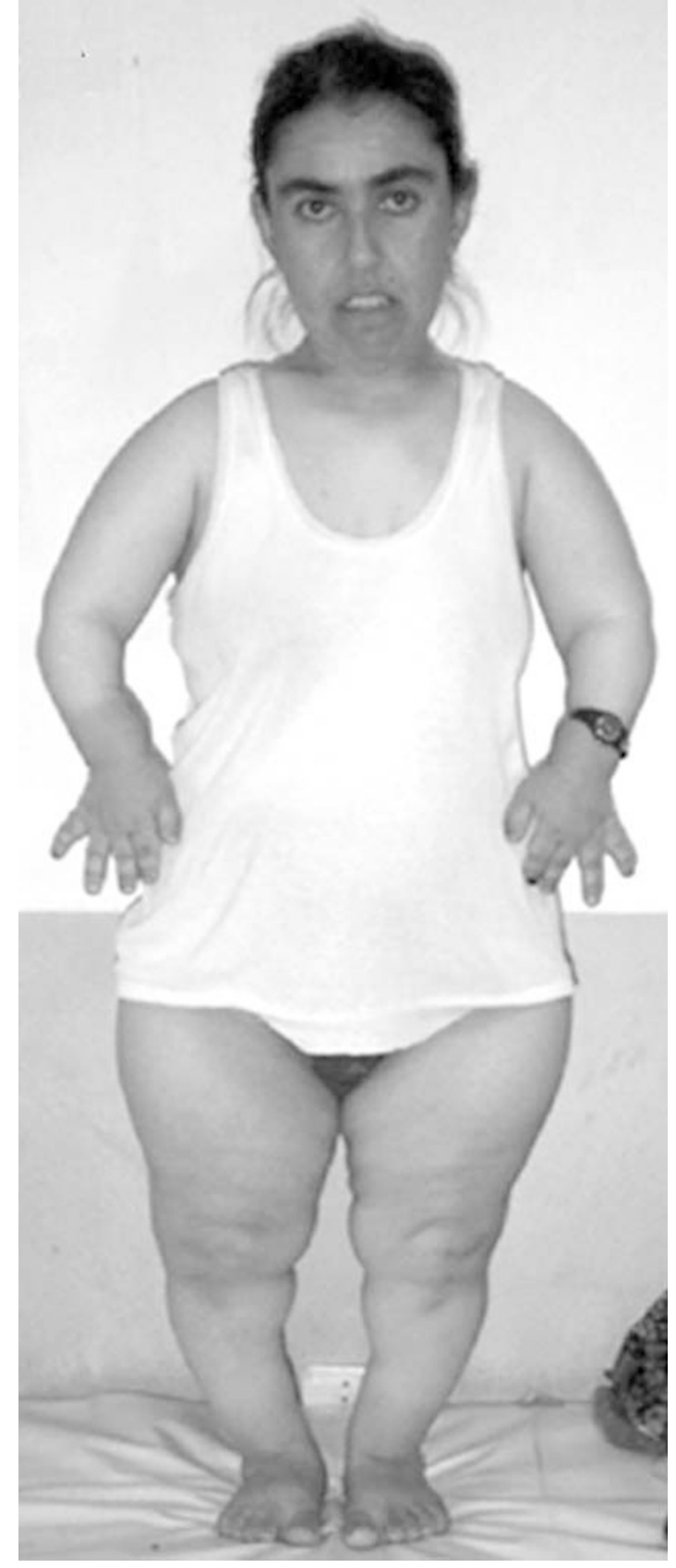

Figure 2 Photograph of proband.

\section{Serum and/or plasma COMP assay}

All women participating in this study as the control group were selected at random (ie, in terms of any given health condition) from the polyclinics of Pamukkale University Hospital and gave informed written consent before blood sampling. Selected participants of the control group were matched to the age range of three patients analyzed for COMP mutations in this study. Three milliliters of peripheral blood were collected into an EDTA-containing tube for plasma separation, and $5 \mathrm{ml}$ of peripheral blood was collected into a plain tube containing no anticoagulant for serum separation. The blood samples were centrifuged at $3000 \mathrm{~g}$ for $10 \mathrm{~min}$, and the plasma and serum were carefully removed from their respective tubes and transferred into plain polypropylene tubes. The samples were stored at $-80^{\circ} \mathrm{C}$ until further processing.

Serum and/or plasma COMP concentrations of 3 patients and 21 controls were analyzed on the basis of an enzyme immunoassay kit (Wieslab, Lund, Sweden), according to the manufacturer's protocol and as described elsewhere. ${ }^{12}$

\section{DNA extraction and COMP mutation detection}

Genomic DNA was extracted from blood samples of all three affected individuals, that is, II-4, II-6, and III-1, using standard methods. Bidirectional fluorescent DNA sequencing was employed to identify mutations in exons $8-19$ of the COMP gene.

\section{Statistical analysis}

Statistical analysis for serum and/or plasma COMP concentrations were performed using the nonparametric Mann-Whitney's $U$-test, and $P<0.05$ was taken as statistically significant.

\section{Results \\ Serum and/or plasma COMP concentrations}

The mean serum and/or plasma COMP concentrations of three affected family members alive $(0.69 \pm 0.15$ and/or $0.81 \pm 0.08 \mu \mathrm{g} / \mathrm{ml}$, respectively) were significantly lower than those of an age-compatible control group of 21 adults $(1.52 \pm 0.37$ and/or $1.37 \pm 0.36 \mu \mathrm{g} / \mathrm{ml}$, respectively; $P<0.0001$; Table 1). There was no overlap in the distribution of serum and/or plasma COMP concentration between PSACH patients and controls (Figure 4).

\section{COMP mutation detection}

All three affected family members were heterozygous for the nucleotide change c.1532A $>\mathrm{G}$ in exon 14 of the COMP gene (Figure 5; nucleotide numbering is according to cDNA sequence with Genbank accession number XM_009336. Nucleotide 1 has been counted as the first nucleotide of the translation initiation codon). This sequence change is predicted to result in a substitution of amino acid 511 from aspartic acid to glycine in COMP (p.Asp511Gly).

\section{Discussion}

Accurate diagnosis of a given osteochondrodysplasia is a time-consuming and often complicated process, involving the clinical and radiographic evaluation of the proband and other family members. Generally, the clinical and radiographic evaluations are used to determine a differential diagnosis before a confirmatory molecular test. This 

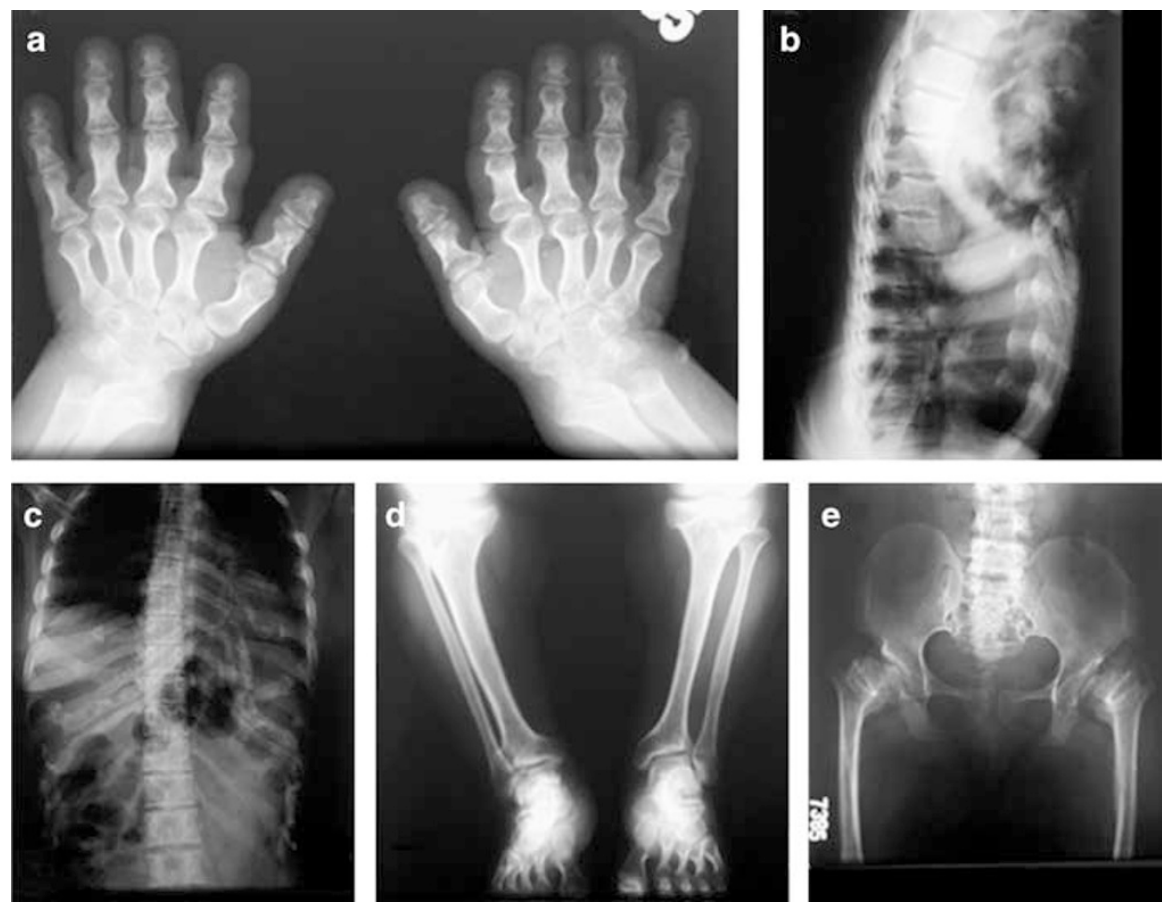

Figure 3 Radiological skeletal survey of the proband showing shortening of long bones and widening in her epiphyses. The hands and feet were small. Phalanges, metacarpals, and metatarsals were short and thick with cone-shaped epiphyses (a and d). The tibiae and fibulae were mildly shortened with splayed metaphyses and epiphyses (d). The shortness of the radii and ulnae was more prominent with widened epiphyses, and mild bowing was present in proximal radii (data not shown). Chest radiogram showed superiorly curved clavicles and spatulate ribs with marked costochondral calcifications (c). Lateral radiograph of the spine revealed straightening of dorsal kyphosis with decreased anteroposterior diameter of the chest, and small central endplate depressions of some dorsal vertebrae (b). There was normal widening of the interpedicular distances from upper to lower spine (b). Pelvis radiogram showed flattened, irregular, and fragmented femoral heads with shortened necks, increased acetabular angles, and widened symphysis pubis (e).

Table 1 COMP concentrations measured in plasma and serum

\begin{tabular}{|c|c|c|c|c|c|c|}
\hline \multirow{2}{*}{$\begin{array}{l}\text { Samples } \\
\text { Group }\end{array}$} & \multirow[b]{2}{*}{$n$} & \multirow[b]{2}{*}{ Age range } & \multicolumn{2}{|c|}{ Plasma COMP $(\mu \mathrm{g} / \mathrm{ml})$} & \multicolumn{2}{|c|}{ Serum COMP $(\mu \mathrm{g} / \mathrm{ml})$} \\
\hline & & & Mean $\pm S D$ & Range & Mean $\pm S D$ & Range \\
\hline $\begin{array}{l}\text { Control } \\
\text { PSACH }\end{array}$ & $\begin{array}{r}21 \\
3\end{array}$ & $\begin{array}{l}24-70 \\
36-82\end{array}$ & $\begin{array}{l}1.37 \pm 0.36 \\
0.81 \pm 0.08^{*}\end{array}$ & $\begin{array}{l}0.95-2.17 \\
0.73-0.88\end{array}$ & $\begin{array}{l}1.52 \pm 0.37 \\
0.69 \pm 0.15^{*}\end{array}$ & $\begin{array}{l}0.97-2.35 \\
0.59-0.87\end{array}$ \\
\hline
\end{tabular}

COMP, cartilage oligomeric matrix protein; $\mathrm{PSACH}$, pseudoachondroplasia.

${ }^{*} P<0.0001$.

study aimed to test the value of serum and/or plasma COMP concentration as a possible diagnostic marker in differentiating PSACH from other osteochondrodysplasias before a more expensive and labor-intensive confirmatory molecular test.

The skeletal survey of the proband revealed a shortlimbed dwarfism with acromesomelic predominance, a generalized epiphyseal and metaphyseal involvement, and uncertain vertebral changes. These radiological findings alone did not allow a clear differentiation between AMDM and PSACH, which were thought to be the most likely diagnoses. The suspected clinical diagnosis of PSACH in the family was supported by decreased serum and/or plasma COMP concentration, and, then subsequently, confirmed by DNA sequencing of COMP and the identification of a potential disease-causing mutation.

The heterozygous nucleotide change (c.1532A $>\mathrm{G})$ in exon 14 of the COMP gene, which is predicted to result in a substitution of amino acid 511 from aspartic acid to glycine has not been published previously. However, it is likely to be the cause of proband's PSACH since aspartic acid 511 is a conserved amino acid in the final repeat of the $\mathrm{Ca}^{2+}$-binding TSP-3 (calmodulin-like) repeat region of COMP. ${ }^{4}$ Furthermore, a mutation of aspartic acid 511 to histidine has been reported to be pathogenic in an unrelated patient with PSACH. ${ }^{13}$ Testing of the affected 

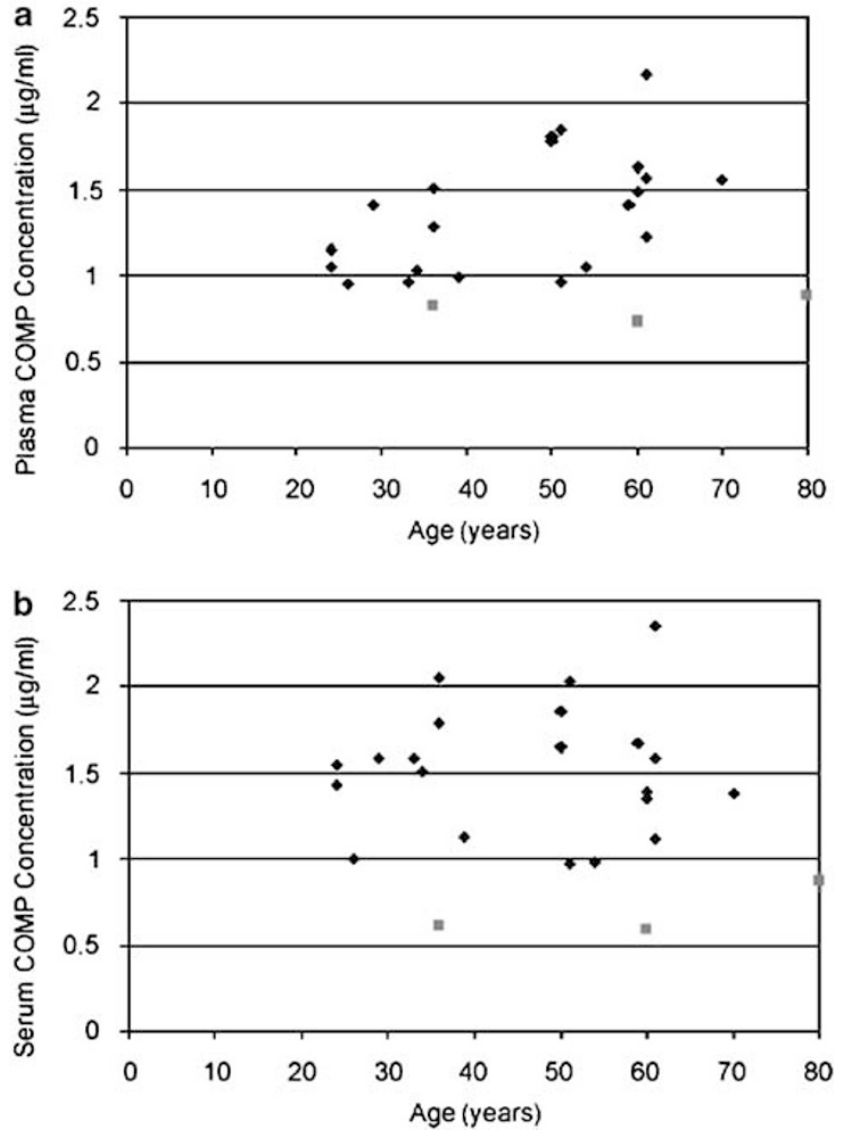

Figure 4 Plasma (a) and serum (b) cartilage oligomeric matrix protein (COMP) concentration distributions of pseudoachondroplasia (PSACH) patients (light squares) compared with controls (dark diamonds).

mother and maternal aunt of the proband, both of whom also tested heterozygous-positive for the nucleotide change $1532 \mathrm{~A}>\mathrm{G}$, is further evidence that this sequence change is likely to be the cause of the proband's PSACH. On the other hand, the pedigree of this family (Figure 1) is very interesting since the proband's grandparents were apparently phenotypically normal. Thus, one would have to postulate significant germline mosaicism for them to have 4 of 7 children affected with an autosomal-dominant disorder.

The diagnosis of PSACH, using DNA sequencing of $C O M P$, is not always easy to accomplish as the mutations can be scattered throughout 12 exons of COMP (exons $8-19) .{ }^{4,14}$ This testing can therefore be expensive and labor-intensive, and is offered in only a few specialized facilities around the world. The average turnaround time for mutation screening of the COMP gene (exons 8-19) by DNA sequencing can be between 2-15 weeks, while the charges from a commercial laboratory may be as high as $€ 1500$. Serum and/or plasma COMP concentration analysis, on the other hand, offers a fast (ie, results are obtained

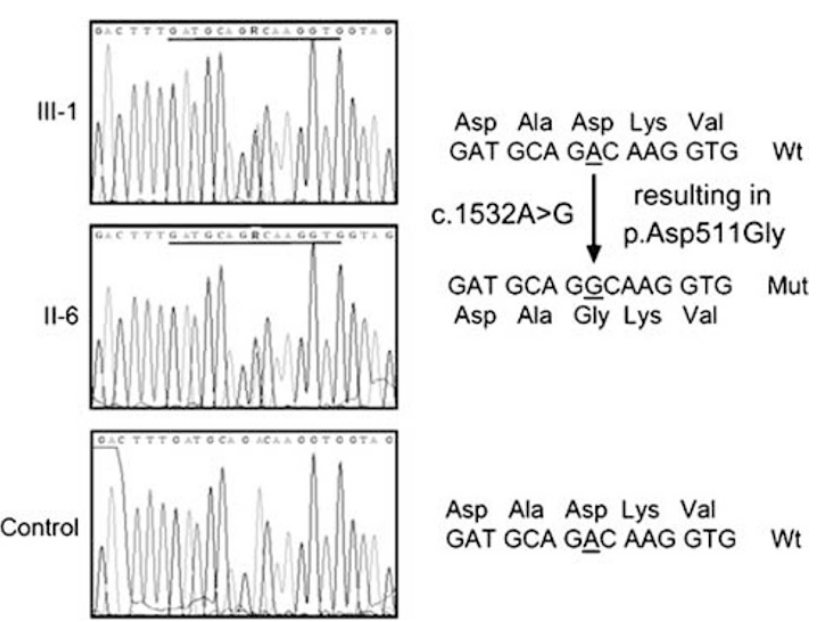

Figure 5 Bidirectional fluorescent DNA sequencing of exons 8-19 of the cartilage oligomeric matrix protein (COMP) gene in all three affected family members revealed the same nucleotide change $(c .1532 \mathrm{~A}>\mathrm{G})$ in exon 14, resulting in a substitution of amino acid 511 from aspartic acid to glycine in COMP (p.Asp511Gly). They were heterozygous for the mutation. Sequence information of individuals III1 and II-6, together with a control, are shown in this figure. Underlined sequences, including the nucleotide change, are detailed at the right. $\mathrm{Wt}$, wild type; Mut, mutated.

the next day) and less expensive (ie, $€ 15$ per testing) alternative that can aid in the selection of patients who are suitable for a confirmatory molecular test.

The study by the manufacturer of the enzyme immunoassay kit for COMP found mean serum concentration of COMP in a group of 80 unselected blood donors (age 2259 ) to be $1.35 \pm 0.40 \mu \mathrm{g} / \mathrm{ml}$ (range: $0.99-2.54$ ). Mabuchi et $\mathrm{al}^{12}$ reported the mean plasma concentration of COMP in a group of 47 control blood donors (age 0-81) as $1.42 \pm 0.35 \mu \mathrm{g} / \mathrm{ml}$ (range: $0.85-2.24$ ). In this study, the mean serum COMP concentration of 21 female-control blood donors (age 24-70) was found as $1.52 \pm 0.37 \mu \mathrm{g} / \mathrm{ml}$ (range: 0.97-2.35), whereas the mean plasma COMP concentration of the same control group was found as $1.37 \pm 0.36 \mu \mathrm{g} / \mathrm{ml}$ (range: $0.95-2.17$ ).

It has been reported that COMP levels in synovial fluid or serum increase significantly in disease conditions, such as rheumatoid arthritis and osteoarthritis, characterized with cartilage destruction. ${ }^{15,16}$ On the contrary, as mentioned earlier, the secretion of mutant COMP from chondrocytes is markedly delayed in PSACH. Thus, low levels of COMP are expected to be found in serum samples of patients with mutations in the COMP gene. Supporting this idea are the findings by Mabuchi et $a l^{12}$ and this presented study. Mabuchi et $a^{12}$ reported the mean plasma COMP concentration of six PSACH patients with COMP mutations as $0.62 \pm 0.13 \mu \mathrm{g} / \mathrm{ml}$ (range: $0.50-0.84$ ), whereas this study reports the mean serum COMP concentration of three PSACH patients with COMP mutations as $0.69 \pm 0.15 \mu \mathrm{g} / \mathrm{ml}$ (range: $0.59-0.87$ ) and the mean plasma 
COMP concentration of the same patients as $0.81 \pm 0.08 \mu \mathrm{g} / \mathrm{ml}$ (range: $0.73-0.88$ ). All these detected decreases were statistically significant $(P<0.0001)$. In addition, Mabuchi et al $^{12}$ reported that the same approach also reveals significant decrease in plasma COMP levels of multiple epiphyseal dysplasia patients carrying COMP mutations.

In conclusion, serum and/or plasma COMP concentration analysis may be suggested as a time and cost-efficient additional diagnostic marker to clinical and radiographic findings in osteochondrodysplasias caused by mutations in the COMP gene.

\section{Acknowledgements}

We thank members of the European Skeletal Dysplasia Network (ESDN) for their advice and help in the clinical and molecular diagnosis of this family. In particular, we thank Michael Briggs and Jacky Taylor (Manchester), Sheila Unger, Andrea Superti-Furga, Jurgen Spranger (Freiburg), Geert Mortier (Gent), Valerie Cormier-Daire (Paris), and Christine Hall (London). The Scientific and Technological Research Council of Turkey (Grant no.: SBAG-104S261) and European Commission (Grant QLG1-CT-2001-02188) supported this study.

\section{References}

1 McKeand J, Rotta J, Hecht JT: Natural history study of pseudoachondroplasia. Am J Med Genet 1996; 63: 406-410.

2 Hecht JT, Nelson LD, Crowder E et al: Mutations in exon 17B of cartilage oligomeric matrix protein (COMP) cause pseudoachondroplasia. Nat Genet 1995; 10: 325-329.

3 Briggs MD, Hoffman SMG, King LM et al: Pseudoachondroplasia and multiple epiphyseal dysplasia due to mutations in the cartilage oligomeric matrix protein gene. Nat Genet 1995; 10: $330-336$.

4 Briggs MD, Chapman KL: Pseudoachondroplasia and multiple epiphyseal dysplasia: mutation review, molecular interactions, and genotype to phenotype correlations. Hum Mut 2002; 19: $465-478$.
5 Hedbom E, Antonsson P, Hjerpe A et al: Cartilage matrix proteins. An acidic oligomeric protein (COMP) detected only in cartilage. J Biol Chem 1992; 267: 6132-6136.

6 Unger S, Hecht JT: Pseudoachondroplasia and multiple epiphyseal dysplasia: new etiologic developments. Am J Med Genet 2001; 106: $244-250$.

7 Rosenberg K, Olsson H, Morgelin M, Heinegard D: Cartilage oligomeric matrix protein shows high affinity zinc-dependent interaction with triple helical collagen. J Biol Chem 1998; 273: 20397-20403.

8 Holden P, Meadows RS, Chapman KL, Grant ME, Kadler KE, Briggs MD: Cartilage oligomeric matrix protein interacts with type IX collagen, and disruptions to these interactions identify a pathogenetic mechanism in a bone dysplasia family. J Biol Chem 2001; 276: 6046-6055.

9 Thur J, Rosenberg K, Nitsche DP et al: Mutations in cartilage oligomeric matrix protein causing pseudoachondroplasia and multiple epiphyseal dysplasia affect binding of calcium and collagen I, II, and IX. J Biol Chem 2001; 276: 6083-6092.

10 Dinser R, Zaucke F, Kreppel F et al: Pseudoachondroplasia is caused through both intra- and extracellular pathogenic pathways. J Clin Invest 2002; 110: 505-513.

11 Newman B, Donnah D, Briggs MD: Molecular diagnosis is important to confirm suspected pseudoachondroplasia. $J$ Med Genet 2000; 37: 64-65.

12 Mabuchi A, Momohara S, Ohashi $\mathrm{H}$ et al: Circulating COMP is decreased in pseudoachondroplasia and multiple epiphyseal dysplasia patients carrying COMP mutations. Am J Med Genet 2004; 129: 35-38.

13 Deere M, Sanford T, Francomano CA, Daniels K, Hecht JT: Identification of nine novel mutations in cartilage oligomeric matrix protein in patients with pseudoachondroplasia and multiple epiphyseal dysplasia. Am J Med Genet 1999; 85: 486-490.

14 Kennedy J, Jackson G, Ramsden S et al: COMP mutation screening as an aid for the clinical diagnosis and counselling of patients with a suspected diagnosis of pseudoachondroplasia or multiple epiphyseal dysplasia. Eur J Hum Genet 2005; 13: 547-555.

15 Mansson B, Carey D, Alini $\mathrm{M}$ et al: Cartilage and bone metabolism in rheumatoid arthritis. Differences between rapid and slow progression of disease identified by serum markers of cartilage metabolism. J Clin Invest 1995; 95: 1071-1077.

16 Sharif M, Saxne T, Shepstone L et al: Relationship between serum cartilage oligomeric matrix protein levels and disease progression in osteoarthritis of the knee joint. $\mathrm{Br} J$ Rheumatol 1995; 34: 306-310 